\title{
Ultrasonic backscattering and microstructure in sheared concentrated suspensions
}

Olivier Lombard, Julien Rouyer, Eric Debieu, Frédéric Blanc, and Emilie Franceschini

Citation: The Journal of the Acoustical Society of America 147, 1359 (2020); doi: 10.1121/10.0000803

View online: https://doi.org/10.1121/10.0000803

View Table of Contents: https://asa.scitation.org/toc/jas/147/3

Published by the Acoustical Society of America

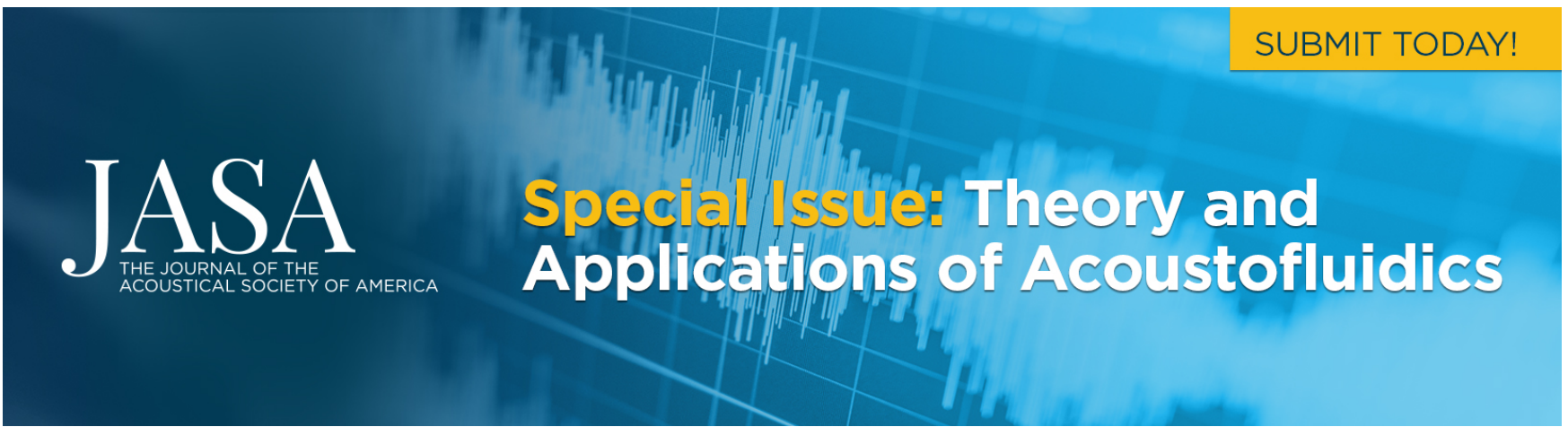




\title{
Ultrasonic backscattering and microstructure in sheared concentrated suspensions
}

\author{
Olivier Lombard, ${ }^{1, a)}$ Julien Rouyer, ${ }^{1}$ Eric Debieu, ${ }^{1}$ Frédéric Blanc, ${ }^{2}$ and Emilie Franceschini ${ }^{1, b}$ \\ ${ }^{1}$ Aix-Marseille Univeristy, CNRS, Centrale Marseille, LMA UMR 7031, Marseille, France \\ ${ }^{2}$ CNRS, Université de Nice, INPHYNI UMR 7010, Nice, France
}

\begin{abstract}
:
Quantitative ultrasound techniques based on the parametrization of the backscatter coefficient (BSC) are used to characterize concentrated particle suspensions. Specifically, a scattering model is fit to the measured BSC and the fit parameters can provide local suspension properties. The scattering models generally assume an isotropic microstructure (i.e., spatial organization) of the scatterers, whereas the sheared concentrated suspensions can develop an anisotropic microstructure. This paper studied the influence of the shear-induced anisotropic microstructure of concentrated suspensions on the ultrasonic backscattering. Experiments were conducted on suspensions of polymethylmetacrylate spheres $(5.8 \mu \mathrm{m}$ in radius $)$ sheared in a Couette flow device to obtain anisotropic microstructure and then mixed by hand to obtain isotropic microstructure. Experimental structure factors that are related to the spatial distribution of sphere positions were obtained by comparing the BSCs of one concentrated and one diluted suspension. Finally, Stokesian dynamics numerical simulations of sheared concentrated suspensions are used to determine the pair correlation function, which is linked to the Fourier transform of the structure factor. The experimental structure factors are found to be in good agreement with numerical simulations. The numerical simulation demonstrates that the angular-dependent BSCs and structure factors are caused by the shear-induced anisotropic microstructure within the suspension. (C) 2020 Acoustical Society of America. https://doi.org/10.1121/10.0000803
\end{abstract}

(Received 25 September 2019; revised 10 January 2020; accepted 9 February 2020; published online 2 March 2020)

[Editor: Keith A. Wear]

Pages: 1359-1367

\section{INTRODUCTION}

Ultrasonic backscattering techniques are used to provide local heterogeneous medium properties (such as scatterer size distribution and/or volume fraction) in optically opaque suspensions. The main fields of application of these techniques are medicine with flowing blood, ${ }^{1,2}$ the food industry, ${ }^{3}$ or the mining industry. ${ }^{4,5}$ In the field of industrial suspensions, the local volume fraction of concentrated suspensions is derived from the local amplitude of the ultrasonic echo envelope or backscatter intensity. ${ }^{4-6}$ In the field of medicine for soft tissue characterization, ultrasonic backscattering techniques are based on parameterizing the Backscatter Coefficient (BSC), which is defined as the power backscattered by a unit volume of scatterers per unit incident intensity per unit solid angle. Specifically, a scattering model is fit to the measured BSC and the fit parameters can provide local tissue properties. ${ }^{2,7}$ A scattering model, called the structure factor model (SFM), has been developed for US characterization of concentrated suspensions of red blood cell aggregates. ${ }^{8,9}$ The SFM considers that at high volume fractions, interference effects are mainly caused by correlations among scatterer positions (i.e., caused by coherent scattering), which are modeled using a

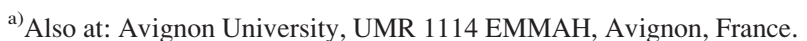

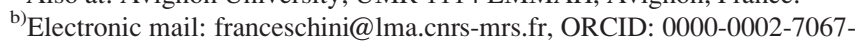
9747.
}

structure factor. ${ }^{10}$ [The structure factor is linked to the Fourier transform of the pair correlation function $g(\boldsymbol{R})$.] Many experimental studies of US blood characterization $^{11-14}$ have focused on the shear-induced disruption of reversible red blood cell aggregates and used the SFM to interpret the backscattering data.

In all the precited works, ${ }^{1-14}$ common assumptions on concentrated suspensions are that (1) the scatterers are much smaller than the wavelength, (2) multiple scattering can be neglected, and (3) the scattering media are homogeneous and isotropic. Little attention has been paid to the influence of shear-induced anisotropic microstructure (i.e., scatterer spatial organization) of suspensions on the US backscattering, which could challenge the third hypothesis of isotropic media. When hard non-Brownian spheres are randomly dispersed in a liquid, the particles are distributed in an isotropic spatial arrangement (a so-called microstructure) which is the result of excluded volume interaction of the particles. However, in an imposed shear flow, this isotropic microstructure is distorted. The anisotropy is more pronounced at close contact between rigid particles. An excess of particles appears on the upstream, where the straining component of the shearing flow drives the particles in contact, whereas there is a lack of particles downstream. Studies conducted in fluid mechanics characterize the microstructure by computing the pair correlation function, $g(\boldsymbol{R})$, which indicates how the particle density varies as a function of position from a reference particle at the origin. The experimental determination of $g(\boldsymbol{R})$ uses non-intrusive methods, such as 
the refractive index matching technique ${ }^{15}$ or X-ray tomography ${ }^{16}$ to explore the microstructure within the bulk of the suspension. The function $g(\boldsymbol{R})$ can also be determined from numerical simulations using the Stokesian dynamics method. ${ }^{15}$ The aforementioned numerical and experimental studies ${ }^{15,16}$ reported shear-induced asymmetric arrangement of the spheres, which is the signature of the existence of nonhydrodynamic contact forces acting between the spheres.

The present study is focussed on shear-induced anisotropic microstructure of non-Brownian concentrated suspensions and its influence on US backscattering. US backscattering measurements were conducted on concentrated suspensions of hard spheres sheared in a Couette flow device. The BSCs were estimated using the reference phantom technique for different insonification angles $\beta$ with respect to the direction of the flow. The structure factors that are related to the spatial distribution of sphere positions were obtained by comparing the BSCs of one concentrated and one diluted suspension. To help to interpret the US measurements, the structure factors were also calculated from the pair correlation function $g(\boldsymbol{R})$ that were determined from Stokesian dynamics numerical simulations. Finally, the numerical structure factors were compared with the experimentally estimated structure factors. The current study contributes to the understanding of US backscattering in sheared concentrated suspensions for improved US characterization of opaque suspensions, such as blood or industrial materials (food, cosmetics, mining).

\section{THEORY: BACKSCATTER COEFFICIENT AND STRUCTURE FACTOR}

We consider a scattering volume $V$ that contains $N$ scatterers insonified by a plane wave of unit pressure amplitude $p_{i}(\mathbf{r})=e^{i \mathbf{k} \cdot \mathbf{r}}$, where $\mathbf{k}=k \mathbf{u}_{i}$ is the wavenumber vector in the direction of propagation of the incident field. By considering far-field regime (i.e., the observation distance is large compared to the size of scattering volume $V$ ) and weak scattering, the field scattered by identical scatterers in the observation point $\mathbf{r}=\mathrm{ru}_{r}$ can be expressed as

$$
p_{s}(\mathbf{r})=\frac{e^{i k r}}{r} f(\mathbf{r}, \mathbf{k}) \sum_{j=1}^{N} e^{i k\left(\mathbf{u}_{i}-\mathbf{u}_{r}\right) \cdot \mathbf{r}_{j}}
$$

where $f(\mathbf{r}, \mathbf{k})$ is the complex scattering amplitude of one single scatterer. The scattered intensity $I_{S}$ is expressed as [see Eq. (11) in Ref. 10]

$$
I_{S}(\mathbf{r}, \mathbf{k})=n V \sigma_{d}(\mathbf{r}, \mathbf{k}) S(\mathbf{r}, \mathbf{k}) / r^{2},
$$

where $n=N / V$ is the number density of scatterers and $\sigma_{d}(\mathbf{r}, \mathbf{k})=|f(\mathbf{r}, \mathbf{k})|^{2}$ is the differential scattering crosssection. The function $S(\mathbf{r}, \mathbf{k})$ is the structure factor linked to the pair correlation function $g(\mathbf{R})$,

$$
S(\mathbf{r}, \mathbf{k})=1+n \int_{V}(g(\mathbf{R})-1) e^{i k\left(\mathbf{u}_{i}-\mathbf{u}_{r}\right) \cdot \mathbf{R}} d^{3} \mathbf{R},
$$

where $g(\boldsymbol{R})$ is related to the density function of the conditional probability $P\left(\boldsymbol{R} \mid \boldsymbol{R}_{\mathbf{0}}=\mathbf{0}\right)$ of finding a sphere with its center at $\boldsymbol{R}$ given that there is a sphere with its center at $\boldsymbol{R}_{\mathbf{0}}=\mathbf{0}$ and the number density of particles $n$ through the equation $g(\boldsymbol{R})=P\left(\boldsymbol{R} \mid \boldsymbol{R}_{\mathbf{0}}=\mathbf{0}\right) / n$. Of particular interest is the backscattering configuration for which $\mathbf{u}_{i}=\mathbf{u}_{r}$. The BSC is expressed as follows:

$$
B S C(\mathbf{k})=\frac{r^{2} I_{s}}{V I_{0}}=n \sigma_{d}(\mathbf{r}, \mathbf{k}) S(\mathbf{k})
$$

where $I_{0}$ is the incident intensity and $S$ is the structure factor in the peculiar case of backscattering configuration given by

$$
S(\mathbf{k})=1+n \int_{V}(g(\mathbf{R})-1) e^{i 2 k \mathbf{u}_{i} \cdot \mathbf{R}} d^{3} \mathbf{R} .
$$

When the scatterers are elastic spheres, the differential scattering cross-section $\sigma_{d}$ in Eq. (4) can be computed by the theory developed by Faran. ${ }^{17}$ The Faran model provides an exact solution for the scattering of sound by a solid sphere in a surrounding fluid medium and therefore includes shear waves in addition to compressional waves. ${ }^{17}$ The BSC expression given by Eq. (4) corresponds to the backscattering model called the SFM. ${ }^{18,19}$

In the case of an isotropic medium, the pair correlation function will only depend on the distance $R$ (and not on the vector $\mathbf{R}$ ), and the structure factor is given by

$$
S(k)=1+2 \pi n \int_{0}^{\infty} \int_{0}^{\pi}(g(R)-1) e^{i 2 k R \sin (\theta)} R^{2} \sin (\theta) d \theta d R .
$$

When considering an isotropic distribution of impenetrable spheres with identical radius $a$, an analytical expression of the structure factor can be obtained by using the Percus-Yevick approximation as established by Wertheim, ${ }^{20}$ and are given by Eqs. (A1)-(A4) in Ref. 19 for backscattering configuration. Some examples of structure factors $S(k)$ are shown in Fig. 1 for several volume fractions $\Phi=1 \%, 10 \%, 30 \%$, and $50 \%$. The structure factors $S$ oscillate around the value of 1 with several peaks. The larger the amplitude of the peaks, the more ordered the scattering medium is. As the product $k a$ increases, the
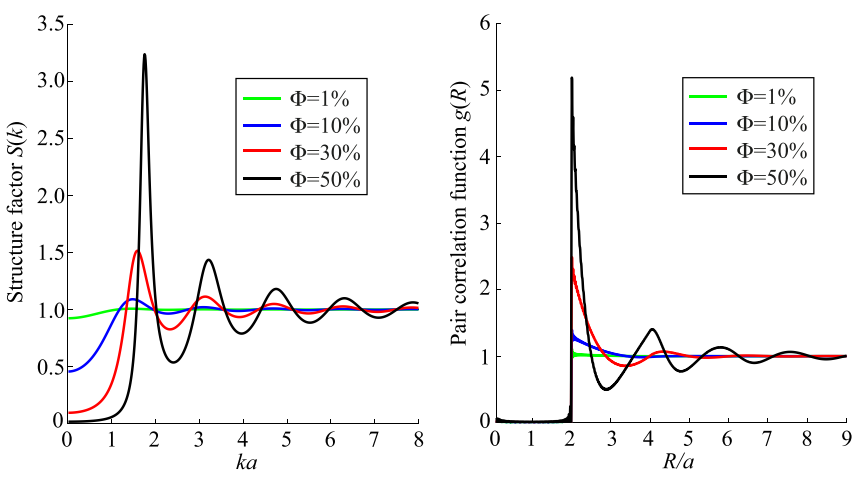

FIG. 1. (Color online) Example of structure factor $S(k)$ and corresponding pair correlation function $g(R)$ for an isotropic distribution of impenetrable spheres with identical radius $a$ for several volume fractions $\Phi=1 \%, 10 \%$, $30 \%$, and $50 \%$. 
oscillation of the peaks becomes weak and $S$ tends to a value of 1. The structure factor gives information on the interference level as a function of $k$ and therefore gives the wavenumbers $k$ that are influenced by the correlation position of particles. If the structure factor is different from the value of 1 , it means that there is a correlation position effect for the considered wavenumber. Also represented in Fig. 1 are the corresponding pair-correlation functions $g(R)$. The function $g$ is zero at short separations $(R<2 a$ ), then $g$ oscillates around the value of 1 with several peaks in the range of $R \geq 2 a$, with a maximum at $R=2 a$.

\section{METHODS}

\section{A. Experimental set-up and ultrasonic data acquisition}

An experimental set-up was designed to combine a Couette flow device with an ultrasonic transducer to probe concentrated suspensions. The suspensions consist of spherical polymethylmethacrylate (PMMA) beads suspended in a Newtonian mixture of water (54 wt. \%) and fructose (46wt. $\%)$. Suspensions of different volume fractions ranging from $1 \%$ to $30 \%$ were used for these experiments. The PMMA microspheres are quasi-monodisperse (CA-10 from Microbeads, Norway) with radius $a=5.8 \pm 0.1 \mu \mathrm{m}$. The size distribution of the microspheres was measured by using optical microscopy (Olympus, IX73), i.e., by detecting the microsphere contour and estimating the radii of 1000 microspheres. The surface roughness of the particles was measured using scanning electron microscopy. Based on measurements on ten particles, the roughness height was found to be around $\epsilon_{R} \approx 16 \times 10^{-3} a$. The density $\rho=1.2 \mathrm{~g}$ $\mathrm{cm}^{-3}$ and Poisson's ratio $\nu=0.35$ of the PMMA microspheres were provided by the manufacturer, and their longitudinal sound speed was measured to be $c=2817 \mathrm{~m} \mathrm{~s}^{-1}$. The composition of the suspending fluid was chosen to closely match the density of the particles at $\rho=1.2 \mathrm{~g} \mathrm{~cm}^{-3}$ and avoid the sedimentation of microspheres. The viscosity and sound speed of the suspending fluid were measured to be $\eta=7.7 \mathrm{mPa} . \mathrm{s}$ and $c_{0}=1729 \mathrm{~m} \mathrm{~s}^{-1}$, respectively.

The experimental set-up is sketched in Fig. 2. The Couette device has a rotating inner cylinder with radius of $40 \mathrm{~mm}$ and height of $50 \mathrm{~mm}$, which is driven by a precision rotating stage (M-0.61.PD from Physik Instrumente, Germany). The outer cylinder is stationary and the gap between the two cylinders is $e=2 \mathrm{~mm}$. All the studied suspensions were sheared at a shear rate of $3.7 \mathrm{~s}^{-1}$. Therefore, in the suspensions, the Reynolds number is small $(\operatorname{Re}=1)$ ensuring a stationary and laminar flow, and the Peclet number $\left(P e \approx 2 \times 10^{4} \gg 1\right.$ ) is large enough to neglect the Brownian motion of particles. The outer stationary cylinder is connected to a water tank where the ultrasonic transducer is immersed (Fig. 2). A hole within the outer stationary cylinder is filled with an agar gel based mixture (water 97.5 wt. \% and agar-agar powder $2.5 \mathrm{wt} . \%$ ) that matches the curvature of the outer cylinder in order to avoid any flow disturbance. The water tank and the agar gel are used to ensure ultrasonic coupling with a fair impedance matching between the
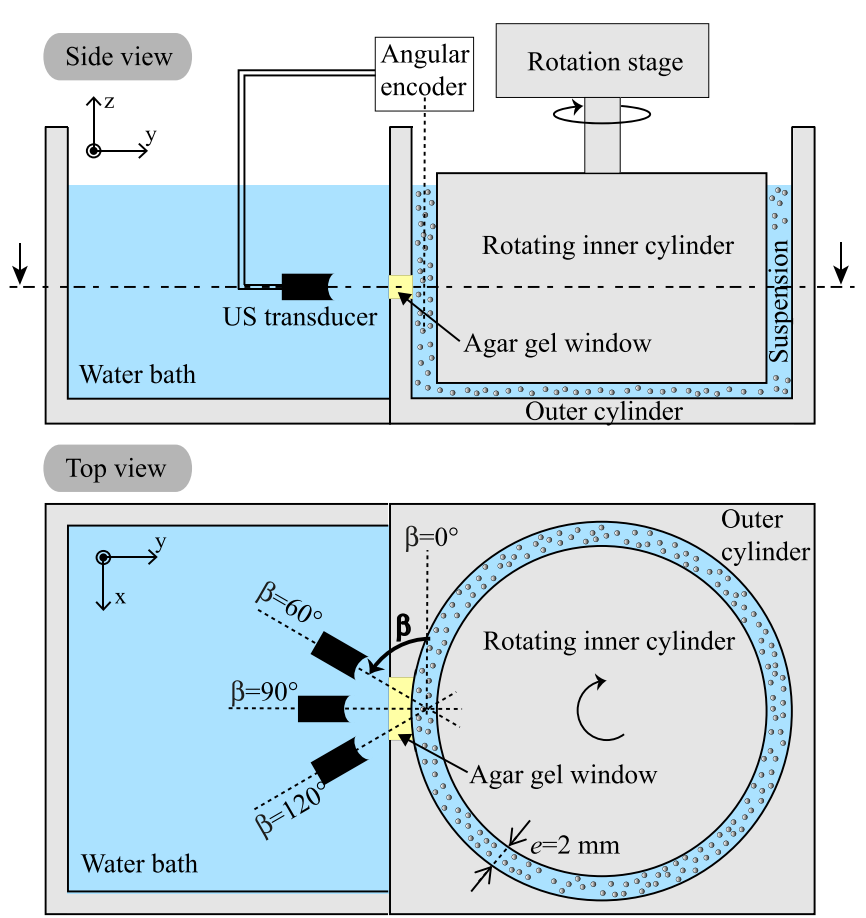

FIG. 2. (Color online) Schematic of the setup, top and side view.

suspension and the ultrasonic transducer. The transducer focus is positioned to have its focal zone at the center of the gap between both cylinders. An angular encoder with a $0.1^{\circ}$ precision (GI333, Baumer, Switzerland) allows us to place the transducer with different insonification angles $\beta$ with respect to the direction of the flow in the Couette device. The insonification angle $\beta$ ranges from $60^{\circ}$ to $120^{\circ}$ with a step of $5^{\circ}$. We used successively two broadband focused transducers with center frequencies of 15 and $30 \mathrm{MHz}$ and focuses of 13.8 and $12.3 \mathrm{~mm}$. The $15-$ and $30-$ $\mathrm{MHz}$ transducers are, respectively, excited by pulserreceiver models 5072 PR and 5073 PR (Olympus, France) and are operating in pulse echo mode. Raw radiofrequency (rf) data are digitized at a sampling frequency of $250 \mathrm{MHz}$, using a high-speed acquisition card (Gagescope, model CS12501, Canada). For each volume fraction $\Phi$ and for each insonification angle $\beta$, around 1000 backscattered rf signals are collected.

The measured $B S C_{\text {meas }}$ was computed using the reference phantom technique with both $15-$ and $30-\mathrm{MHz}$ transducers. $^{21}$ The reference phantom technique is used to account for the electromechanical system response and the depth-dependent diffraction and focusing effects of the ultrasound beam. ${ }^{21}$ The reference sample is a suspension at a low volume fraction of $1 \%$, which consists of monodisperse PMMA microspheres of radius $2.8 \mu \mathrm{m}(\mathrm{CA}-06$ from Microbeads, Norway) suspended in a Newtonian mixture of water (54 wt. \%) and fructose (46wt. \%). The reference sample was sheared in the Couette flow device and the ultrasonic acquisitions were performed for the thirteen insonification angles $\beta$, as done previously for the $\mathrm{CA}-10$ particle suspensions. The use of the reference sample within the 
Couette flow device allows us to account for attenuation and transmission losses caused by the agar layer. Backscattered signals are selected at the central focal zone of the transducer with a rectangular window of axial length corresponding to 12 wavelengths for both studied suspensions and reference sample. The power spectra of 1000 windowed echoes are averaged to obtain $S_{s}$ and $S_{\text {ref }}$. The measured $B S C_{\text {meas }}$ was computed as follows:

$$
B S C_{\text {meas }}(f)=\frac{S_{s}(f, z)}{S_{r e f}(f, z)} B S C_{r e f}(f) e^{2\left(\alpha_{s}(f)-\alpha_{r e f}(f)\right) 2 d},
$$

where $B S C_{r e f}$ is given by the SFM model using Eq. (4). The exponential term in Eq. (7) corresponds to the compensation for the relative attenuation of the suspension in comparison with the reference sample $\left[\alpha_{s}(f)-\alpha_{r e f}(f)\right]$, that was determined as described below. The value $2 d$ corresponds to the propagation distance in the suspension.

For each studied suspension, the relative attenuation $\left[\alpha_{s}(f)-\alpha_{r e f}(f)\right]$ was measured with the $30 \mathrm{MHz}$ transducer in the reflection mode at the fixed angle $\beta=90^{\circ}$. The rotating inner cylinder was used as the reflector. Acquisitions of $200 \mathrm{rf}$ signals were performed both with suspension under consideration and with reference sample in the Couette flow device. The relative attenuation $\left[\alpha_{s}(f)-\alpha_{\text {ref }}(f)\right]$ was determined using an insertion-loss broadband technique. The measured relative attenuation (expressed in $\mathrm{Np} / \mathrm{mm}$ ) was fitted with the function $\alpha_{0} f$, where $f$ is the frequency in $\mathrm{MHz}$. This fitting curve was valid in the frequency range 15-30 MHz bandwidth for all studied suspensions. The values of $\alpha_{0}$ were found equal to $0.04,0.12$, and $0.28 \mathrm{~Np} / \mathrm{mm} /$ $\mathrm{MHz}$ for suspensions of volume fractions $10 \%, 20 \%$, and $30 \%$, respectively.

To obtain the experimental structure factor, two measurements of suspensions with identical PMMA microbeads and with two different volume fractions are required: a lower volume fraction for a reference purpose and another volume fraction under consideration. ${ }^{19,22}$ Note that the lower volume fraction is chosen to be sufficiently low such that the structure factor is assumed to be unity (incoherent scattering). The structure factor was obtained experimentally by ${ }^{19}$

$$
S_{\text {meas }}(k)=\frac{\Phi_{L} B S C_{\text {meas }}(k)}{\Phi B S C_{\text {meas }, L}(k)},
$$

where $k$ is the wavenumber, $\Phi_{L}$ and $B S C_{\text {meas, } L}$ are the volume fraction and the measured BSC for the lower volume fraction studied (equal to $1 \%$ ), and $\Phi$ and $B S C_{\text {meas }}$ represent the volume fraction and the measured BSC for the volume fraction under consideration.

All the experimental procedures were repeated twice with both transducers for each studied suspension.

\section{B. Numerical simulations}

We compute the structure factors from numerical simulations performed by Blanc et al. ${ }^{15}$ that provide the position of spheres in sheared concentrated suspensions. The numerical procedure used in Blanc et al. ${ }^{15}$ (Sec. III) is briefly recalled here. The Stokesian dynamics is used to simulate the flow of a suspension of spheres in a viscous fluid submitted to an imposed shear at zero Reynolds number. A monodisperse system of 125 spheres is considered in a cubic box $V_{s}$ with periodic boundaries. The Brownian motion is neglected in the computation by considering a large Peclet number $\left(P e \approx 10^{9}\right)$. A repulsive force between the spheres is also introduced to consider the particle roughness (approximately equal to $\epsilon_{R} \approx 3 \times 10^{-3} a$ ). For a given volume fraction, 250 cubic boxes are simulated to accumulate enough particle spatial positioning for the computation of pair correlation function $g(\mathbf{R})$.

The procedure to compute $g(\mathbf{R})$ is briefly summarized here. First, a volume $V_{r}=L^{3}$ is defined as a cubic box of edge $L=6 a$ at the center of $V_{s}$. The volume $V_{r}$ contains the reference particles (1). From the center of each reference particle, the position vectors of all other particles (2) situated in $V_{s}$ are computed, and the separating vector between particle centers is denoted by $\mathbf{R}_{12}=\left(R_{12}, \theta_{12}, \psi_{12}\right)$, where $\theta$ and $\psi$ denote the colatitude and the longitude angles, respectively. Note that the top view in Fig. 2 corresponds to $\theta$ $=\pi / 2$ and $\psi=0$ is the direction of the flow. The $(R, \theta, \psi)$ is sampled as $\Delta R=a / 100, \Delta \theta=\pi / 10 \mathrm{rad}$, and $\Delta \psi=\pi / 40 \mathrm{rad}$. The number $N_{p}(R, \theta, \psi)$ of pair separation vectors in the volume $\quad(R-\Delta R / 2, R+\Delta R / 2 ; \quad \theta-\Delta \theta / 2, \theta+\Delta \theta / 2$; $\psi-\Delta \psi / 2, \psi+\Delta \psi / 2)$ is determined. Finally, the pair correlation function is computed as

$$
g(R, \theta, \psi)=\left\langle\frac{1}{n} \frac{N_{p}(R, \theta, \psi)}{N_{1} \sin (\theta) R^{2} \Delta R \Delta \theta \Delta \psi}\right\rangle,
$$

where $N_{1}$ is the number of reference particles (1) and \langle\rangle is the mean over 250 cubic boxes. To predict the structure factor that can be measured by ultrasound, the structure factor $S(\mathbf{k})$ was computed as a function of the wave number $k$ and the direction of the incident wave given by $\beta$ as

$$
\begin{aligned}
S(k, \beta)= & 1+n \int(g(R, \theta, \psi)-1) \\
& \times e^{2 j k R \sin (\theta) \cos (\beta-\psi)} R^{2} \sin (\theta) d \theta d \psi d R .
\end{aligned}
$$

Note that within the simulation box of $L^{3}$, the structure factor is unreliable for $k<\pi / L$, so the structure factor can be only computed for the lowest $k a \approx 0.5$ (here $L=6 a$ ).

\section{RESULTS}

\section{A. US measurements from non sheared suspensions}

US measurements were performed by simply mixing by hand the suspension at $\Phi=10 \%$ with a thin stick of diameter less than $1.5 \mathrm{~mm}$. After each mixing, a single backscattered signal was acquired. Because of the consuming time to manually acquire the signals, we collect 200 backscattered signals (instead of 1000) from two insonification angles $\beta=90^{\circ}$ and $120^{\circ}$. With this simple procedure, the aim was 

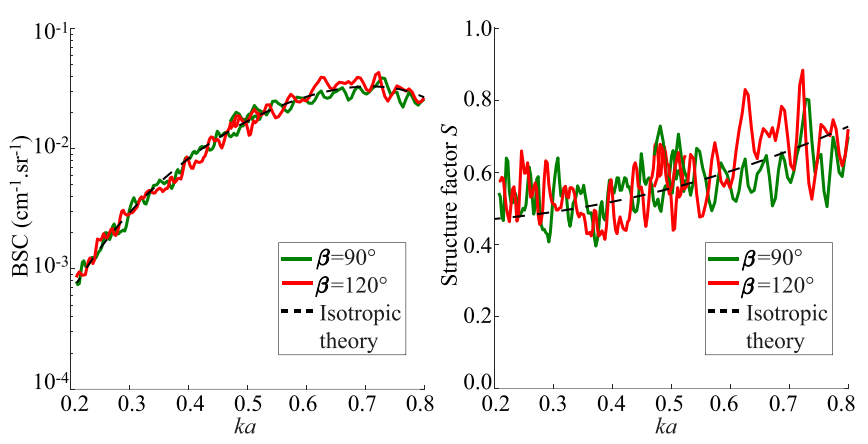

FIG. 3. (Color online) Measured $B S C_{\text {meas }}$, and corresponding structure factors $S_{\text {meas }}$ (solid lines) from a suspension mixed by hand $(\Phi=10 \%)$. Measurements were performed for two insonification angles $\beta=90^{\circ}$ and $120^{\circ}$.

to prepare a non sheared suspension with a homogeneous and isotropic microstructure.

Figure 3 shows the measured $B S C_{\text {meas }}$, and the structure factors $S_{\text {meas }}$ from the suspension mixed by hand. Both $B S C_{\text {meas }}$ and $S_{\text {meas }}$ are plotted as a function of the product $k a$ to facilitate comparison between experiments and simulations, as shown later in Sec. IV C. The range $k a=[0.20-0.52]$ corresponds to the frequency bandwidth [10-25] $\mathrm{MHz}$ probed with the $15-\mathrm{MHz}$ transducer and the range $k a=[0.48-0.80]$ corresponds to the frequency bandwidth [23-38] MHz probed with the $30-\mathrm{MHz}$ transducer.

We can observe in Fig. 3 that the $B S C_{\text {meas }}$ and $S_{\text {meas }}$ are similar for the two insonification angles $\beta=90^{\circ}$ and $120^{\circ}$, which confirms that the suspension mixed by hand has an isotropic microstructure.

\section{B. US measurements for sheared concentrated suspensions}

Some examples of measured $B S C_{\text {meas }}$, and structure factors $S_{\text {meas }}$ are presented in Figs. 4(a) and 4(b) for sheared suspensions at volume fractions $\Phi=1 \%, 10 \%$ and $30 \%$. Note that the structure factor cannot be computed for the suspension with the lowest volume fraction of $1 \%$, since the $B S C_{\text {meas }, L}$ for the lower volume fraction is used in the denominator of Eq. (8) to compute the structure factor. Important amplitude variations of both $B S C_{\text {meas }}$ and $S_{\text {meas }}$ can be observed depending on the insonification angles $\beta$ for volume fractions of $10 \%$ and $30 \%$. The relative differences between $B S C_{\text {meas }}\left(\beta=60^{\circ}\right)$ and $B S C_{\text {meas }}\left(\beta=90^{\circ}\right)$ averaged over all frequencies are equal to 3.1 and $3.9 \mathrm{~dB}$ for volume fractions of $10 \%$ and $30 \%$, respectively, whereas this relative difference averaged over all frequencies is equal to $0.4 \mathrm{~dB}$ for the smallest volume fraction of $1 \%$ so that the sheared suspension of $1 \%$ was considered to have isotropic microstructure.

For comparison purposes, the theoretical predictions of $B S C_{\text {theo }}$ and $S_{\text {theo }}$ using the SFM modeling in the isotropic case are represented in Figs. 4(a) and 4(b) (see black dashed lines). More precisely, $B S C_{\text {theo }}$ was calculated using Eq. (4) and $S_{\text {theo }}$ using Eqs. (A1)-(A4) in Ref. 19. The majority of
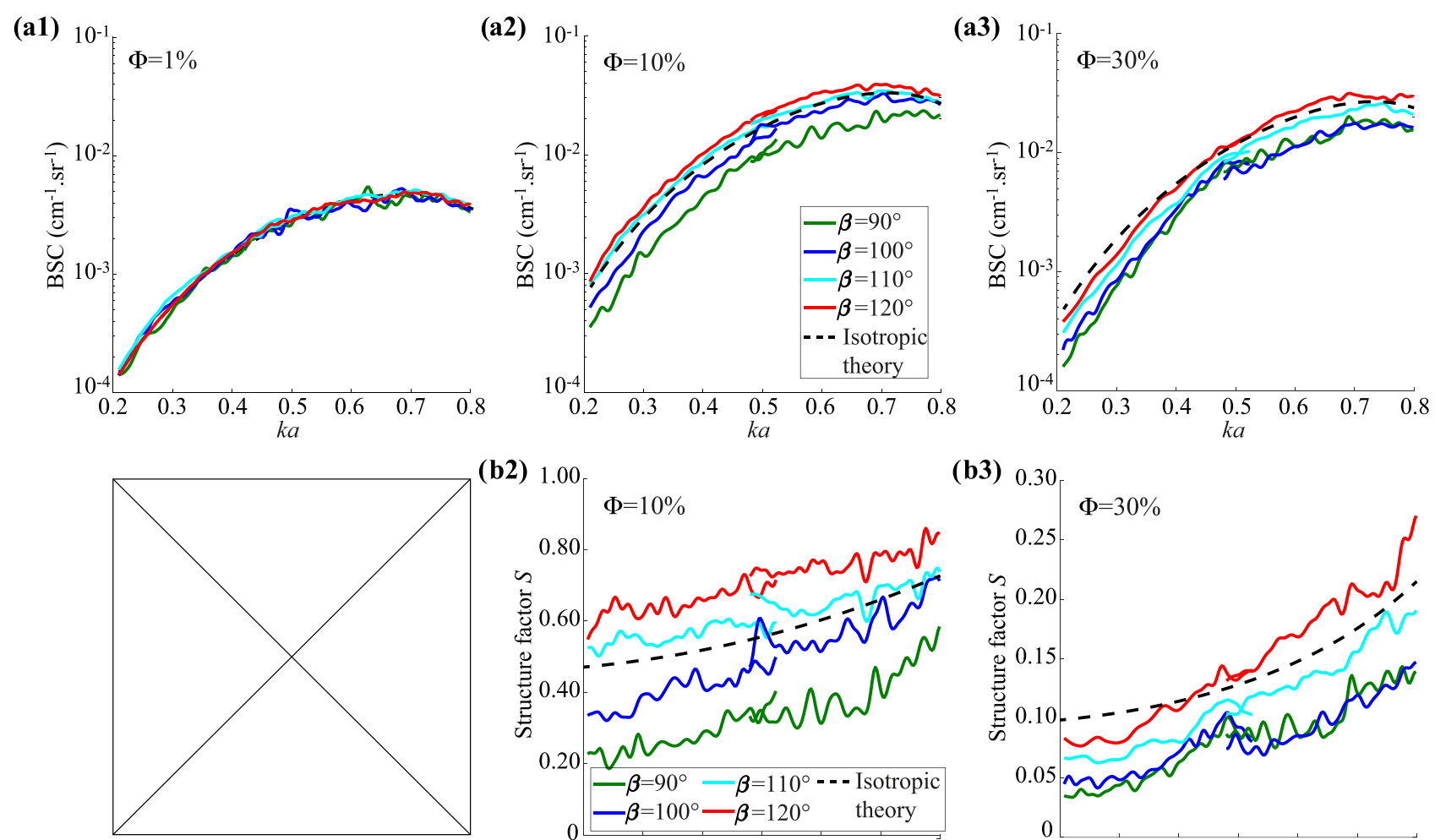

(b2)
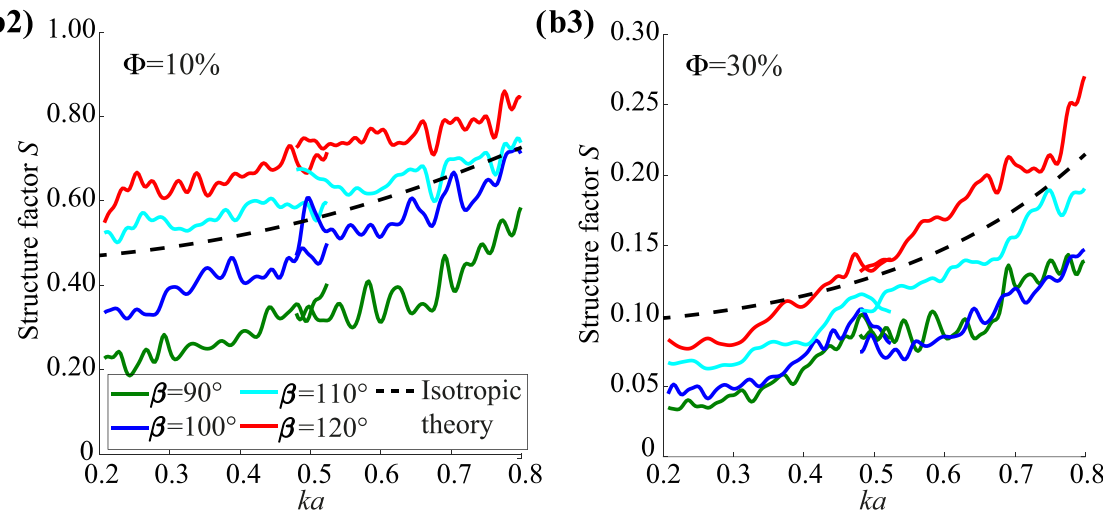

FIG. 4. (Color online) (a) Measured $B S C_{\text {meas }}$, as a function of the product $k a$ (solid lines) from suspensions sheared in the Couette device at various insonification angles $\beta$ for volume fractions $\Phi=1 \%, 10 \%$, and $30 \%$. Also represented are the theoretical $B S C_{\text {theo }}$ computed by the SFM in the isotropic case (dashed lines). (b) Same as (a) for the structure factor as a function of the product $k a$. Note that the structure factor at $\Phi=1 \%$ is not represented because it cannot be computed for the lowest volume fraction of $1 \%$. 
theoretical and experimental curves differ strongly in terms of amplitude and frequency dependence. However, some theoretical and experimental BSCs have similar frequency dependence, as it can be observed by fitting the BSC with a power law of the form $b f^{m}$ within the $10-38 \mathrm{MHz}$ frequency bandwidth. For example, at $\Phi=10 \%$, the exponents $m$ of the fitted $B S C_{\text {meas }}$ curve for $\beta=110^{\circ}-120^{\circ}(m=2.81)$ are similar to that of the isotropic theoretical $B S C_{\text {theo }}(m=2.85)$ [see also the red and cyan curves in Fig. 4(a2)].

\section{Simulations results for sheared suspensions}

Figure 5(a) displays examples of the two-dimensional (2D) map of the pair correlation function $g$ in the plane of shear from numerical simulations, as shown previously in Fig. 6 of Blanc et al. ${ }^{15}$ The pair correlation functions are anisotropic with regions depleted in particles and regions with high particle concentration. The direction of the depleted area is situated around the angle $\psi=5^{\circ}$ (i.e., close to the velocity direction) for $\Phi=10 \%$, and around the angle $\psi=15^{\circ}$ for $\Phi=30 \%$. The high particle concentration area is symmetric with respect to the axis $\psi=0^{\circ}$ for $\Phi=10 \%$ and with respect to the axis $\psi=15^{\circ}$ for $\Phi=30 \%$. For $\Phi=10 \%$, the symmetrical axis of the high particle concentration area differs slightly from the direction of the depleted area, the pair correlation function at $\Phi=10 \%$ thus has a pseudo-symmetrical axis in a direction between $\psi=0^{\circ}$ and $\psi=5^{\circ}$. For $\Phi=30 \%$, the direction of the symmetrical axis is the same as the direction of the depleted area. The pair correlation function at $\Phi=30 \%$ has a pseudo-symmetrical axis in the direction $\psi=15^{\circ}$ [pseudo-symmetrical axis are noted as dashed lines in Fig. 5(a)]. Indeed, the pair correlation function is fore-and-aft asymmetric, with a tail-like
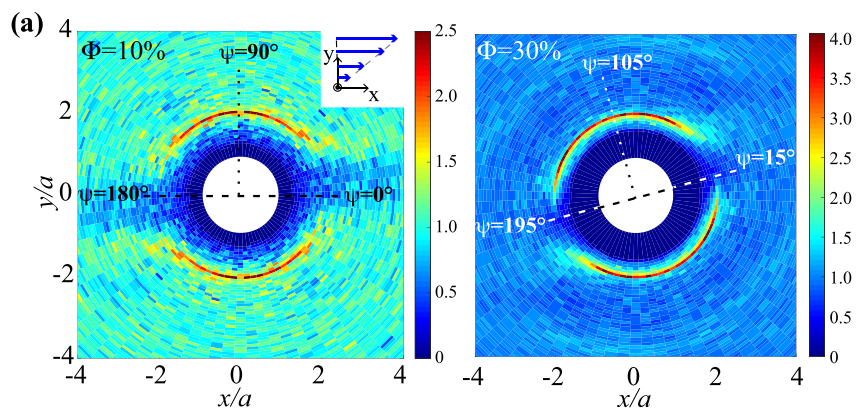

(b)

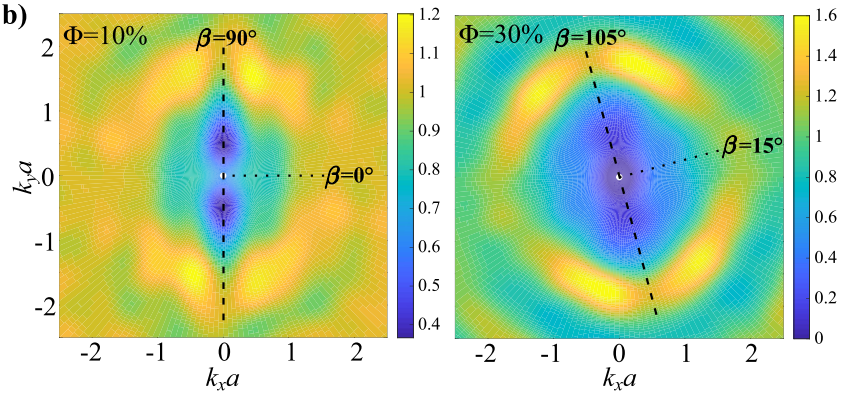

FIG. 5. (Color online) (a) Numerical pair correlation function $g(\mathbf{r})$ in the shear plane $(\theta=\pi / 2)$ for volume fractions $\Phi=10 \%$ and $30 \%$. (b) Corresponding structure factor $S(\mathbf{k})$. The dashed lines indicate the pseudo symmetrical axis.
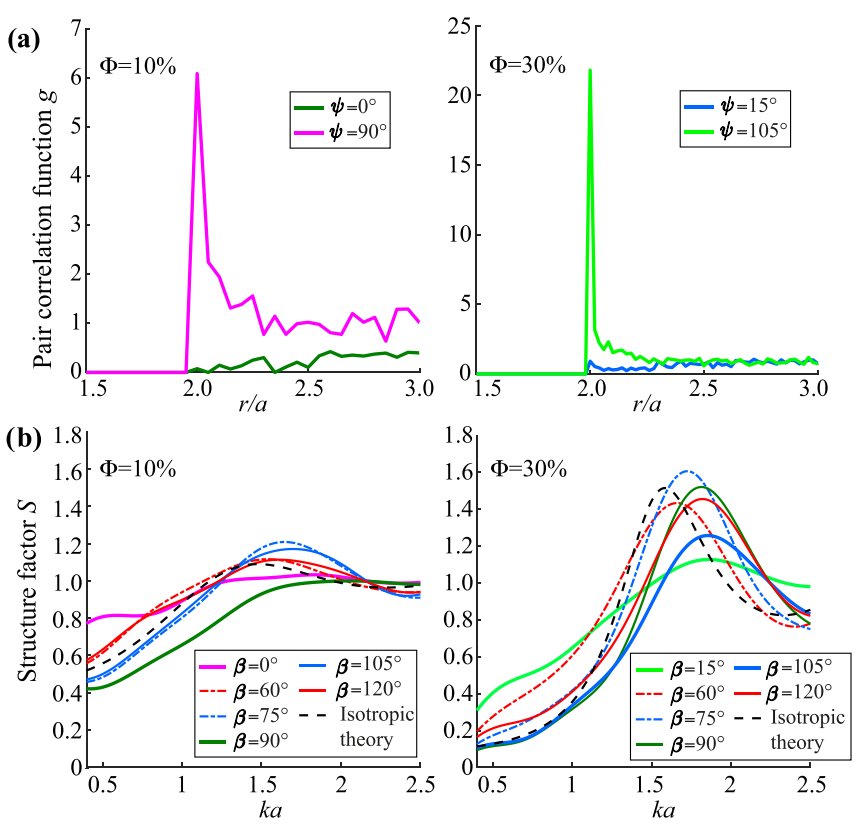

FIG. 6. (Color online) (a) 1D scalar plots of the numerical pair correlation function $g$ for volume fractions $\Phi=10 \%$ and $30 \%$. (b) Numerical structure factors $S$ at various insonification angles $\beta$ for volume fractions $\Phi=10 \%$ and $30 \%$.

high-particle concentration zone observed in the recession quadrant but not in the approach quadrant. ${ }^{23}$ That is why we use the term pseudo-symmetrical axis instead of symmetrical axis to describe this asymmetric effect.

The anisotropy of the pair correlation function comes with an anisotropic structure factor, as it can be observed on the 2D maps of the corresponding structure factors in Fig. 5(b). The structure factor depends on $\left(k_{x}, k_{y}\right)$ that is in the dual space of $(x, y)$. There is a $90^{\circ}$ rotation angle between the pseudo-symmetrical axis in the real space $(x, y)$ and its associated pseudo-symmetrical axis in the dual space $\left(k_{x}, k_{y}\right)$. For $\Phi=10 \%$ (and $\Phi=30 \%$, respectively), the pair correlation function has its pseudo-symmetrical axis around $\psi=0^{\circ}-5^{\circ}$ (and $\psi=15^{\circ}$, respectively), and the corresponding structure factor has its pseudo-symmetrical axis around $\beta=90^{\circ}$ in the dual space (and $\beta=105^{\circ}$, respectively). Finally, the structure factor is asymmetric as the pair correlation function. Some examples of the one-dimensional (1D) scalar plots for both $g$ and $S$ are shown in Fig. 6 for volume fractions $\Phi=10 \%$ and $\Phi=30 \%$ at different angles.

Figure 7 displays the angular variation of the structure factor obtained from numerical simulations of sheared suspensions at $k a=0.8$ (solid lines) and $k a=1.7$ (dashed lines). For the studied volume fractions, the structure factors $S(k a=0.8)$ versus insonification angle $\beta$ share a similar behavior: the $S$ amplitude is quasi-constant for $0^{\circ} \leq \beta$ $\leq 60^{\circ}$, and then the $S$ curve has a U-shape for $60^{\circ} \leq \beta$ $\leq 120^{\circ}$. For a fixed insonification angle, the amplitude of the structure factor $S$ decreases at $k a=0.8$ and increases at $k a=1.7$ with increasing volume fraction. These changes (i.e., the decrease for $k a=0.8$ and the increase for $k a=1.7$ ) in $S$ amplitude are also obtained in the case of isotropic 


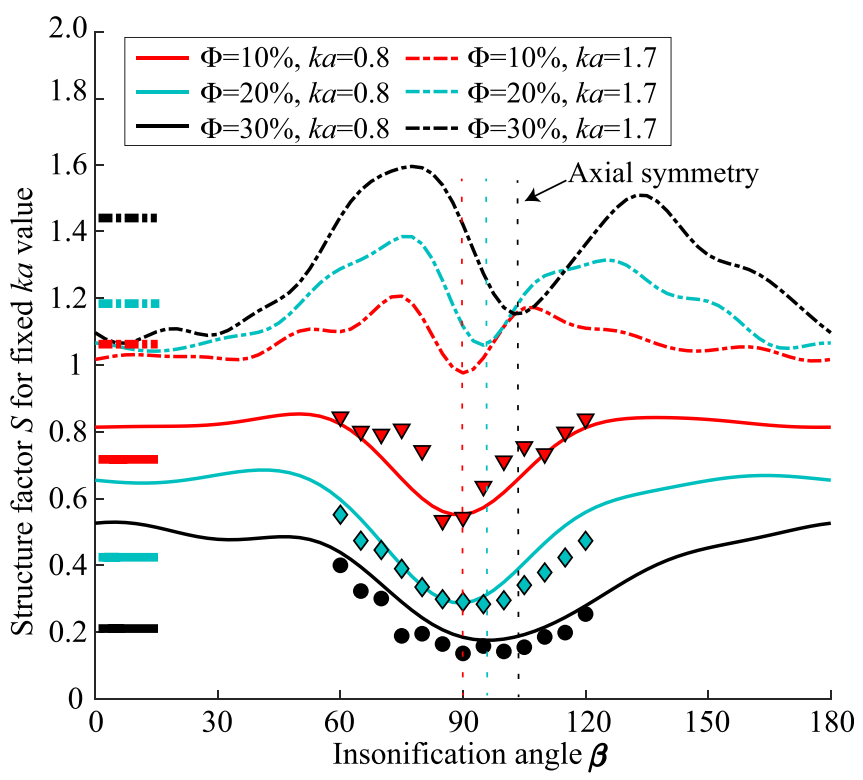

FIG. 7. (Color online) Structure factor at fixed value of $k a$ as a function of the insonification angle $\beta$ for volume fractions $\Phi=10 \%, 20 \%$, and $30 \%$. The thin solid and dashed lines represent the structure factors obtained from the Stokesian dynamics numerical simulations for $k a=0.8$ and $k a=1.7$, respectively. The thick solid and dashed lines on the left side represent the theoretical $B S C_{\text {theo }}$ computed by the SFM in the isotropic case for $k a=0.8$ and $k a=1.7$, respectively. The symbols represent the structure factors measured by ultrasound $S_{\text {meas }}(k a=0.8)$.

media, as it can be observed with the thick solid and dashed lines of theoretical $B S C_{\text {theo }}$ in the isotropic case in Fig. 7. This is caused by a closer packing as the volume fraction increases, leading to a greater orderliness in the particle spatial arrangement. Also given in Fig. 7 are the measured structure factor $S_{\text {meas }}(k a=0.8)$ as a function of the insonification angle $\beta$ (see symbols) for one series of experiments. Overall, the structure factor measurements are in good agreement with the numerical simulations. All the experimental procedure was repeated twice (i.e., the preparation of suspensions at various volume fractions and the corresponding ultrasonic measurements) to verify the measurement repeatability. The angular variation of $S_{\text {meas }}(k a=0.8)$ obtained from the two series of experiments are given in the supplemental figure. ${ }^{28}$ The $S_{\text {meas }}(k a=0.8)$ measured in the two studies have similar amplitude, showing good repeatability.

\section{DISCUSSION AND CONCLUSION}

\section{A. Origin of the anisotropic structure factors}

In the present study, structure factors were computed from the Stokesian dynamics numerical simulations of sheared concentrated suspensions. The anisotropy of the numerical structure factors is clearly caused by the shearinduced anisotropic microstructure within the suspension. This anisotropic microstructure is related to contact perturbations due to roughness particle as explained in the following. For perfectly smooth spheres, the relative trajectories of two interacting particles are reversible and symmetric (see Guazzelli and Pouliquen, ${ }^{24}$ Fig. 5, left panel). Particles are kept apart by lubrification, and the compressive and extensional regions of the flow, aft and fore, respectively, cancel out. Whereas for rough particles, the trajectories are irreversible and asymmetric (see Guazzelli and Pouliquen, ${ }^{24}$ Fig. 5, right panel). When the rough particles are driven so close to one another by the shear flow with a minimum approach distance smaller than the roughness, the spheres enter into contact in the compressional region of the flow and move apart in the extensional region leading to a region depleted in particles. As the volume fraction increased, the pair correlation function $g$ becomes more peaked at close contact (near $r / a \approx 2$ ), and the depleted zone that is close to the velocity direction at $\Phi=10 \%$ rotates toward the dilatation axis direction at $\Phi=30 \%$ [Fig. 5(a)].

For the angles comprised between $\psi=45^{\circ}$ and $\psi=135^{\circ}$ at $\Phi=10 \%$, and between $\psi=60^{\circ}$ and $\psi=150^{\circ}$ at $\Phi=30 \%$, the pair correlation function [plotted in Fig. 6(a)] behaves as the pair correlation function of an isotropic distribution of spheres (plotted in Fig. 1). Specifically, the $g$ is zero at short separations $(r / a<2)$, then $g$ oscillates around the value of 1 with a large peak around $r / a \approx 2$. The isotropic behavior of $g$ is accompanied by the smallest dynamic range of the structure factor in the angular direction $\beta=0^{\circ}$ for $\Phi=10 \%$ and $\beta=15^{\circ}$ for $\Phi=30 \%$ (see Fig. 6). These angular directions correspond to the orthogonal directions for which the pair correlation function has an isotropic behavior and the medium is less ordered.

The angular position of the depletion zone is comprised between $\psi=-15^{\circ}$ and $\psi=15^{\circ}$ for $\Phi=10 \%$ and between $\psi=0^{\circ}$ and $\psi=30^{\circ}$ for $\Phi=30 \%$, as it can be observed on the pair correlation functions shown in Figs. 5(a) and 6(a). The depletion zone corresponds to higher exclusion distance between particles. Specifically, within the depletion zone, the $g$ has no peak at close contact (near $r / a \approx 2$ ) and its amplitude remains much lower than the value of 1 for $r / a>2$. This $g$ behavior means that the main axis of the depleted area (at $\psi=0^{\circ}$ for $\Phi=10 \%$ and at $\psi=15^{\circ}$ for $\Phi=30 \%$ ) corresponds to a high degree of ordering. This higher ordering goes with a large dynamic range of $S$ for the angles comprised between $\beta=60^{\circ}$ and $\beta=120^{\circ}$ at $\Phi=10 \%$, and between $\beta=60^{\circ}$ and $\beta=150^{\circ}$ at $\Phi=30 \%$, as shown in Figs. $5(\mathrm{~b})$ and $6(\mathrm{~b})$. As mentioned previously, the angular direction of a large dynamic range of $S$ corresponds to the orthogonal direction of the main axis of the depleted area.

It is also interesting to focus on the pair correlation function at long distances $r$ in order to gain a better understanding of the structure factor at low $k a$ values in Fig. 5. The $g$ exhibits a large angle-dependent amplitude in the interval $2<r / a<4$ for $\Phi=10 \%$, whereas the $g$ is nearly isotropic in the interval $2.4<r / a<4$ for $\Phi=30 \%$. The $g$ at $\Phi=10 \%$ has variations of angle-dependent amplitude over a larger interval of distance than the $g$ at $\Phi=30 \%$. This explains why the amplitude of the structure factor at low $k a$ values $(<1)$ depends more on the angle for $\Phi=10 \%$ than for $\Phi=30 \%$.

The angular-dependent structure factors obtained from numerical simulations of sheared suspensions are plotted in 
Fig. 7. At $k a=1.7$, the angular-dependent $S$ curve has a pseudo symmetrical axis around the angles $\beta=90^{\circ}, 95^{\circ}$, and $102^{\circ}$ at $k a=1.7$, respectively, for $\Phi=10 \%, 20 \%$, and $30 \%$. This angular direction corresponds to the orthogonal direction of the axis of the depleted area of the pair correlation function. (The reader can refer to Blanc et al., ${ }^{15}$ Fig. 9, for the evolution of the rotation of the 2D pair correlation function $g$ with the particle volume fraction.) It is interesting to observe that the angular-dependent $S_{\text {meas }}$ measured by ultrasound has also pseudo symmetrical axis around the angles $\beta=90^{\circ}, 95^{\circ}$, and $100^{\circ}$, respectively, for $\Phi=10 \%$, $20 \%$, and $30 \%$ (Fig. 7). It means that the angular position of the main depletion zone can be detected by the structure factor measurements at low $k a$ values.

\section{B. Isotropic and anisotropic ultrasonic measurements}

To reinforce our demonstration that the anisotropic ultrasonic measurements originate from the shear-induced anisotropic microstructure within the suspension, experiments were also conducted with a suspension at volume fraction $\Phi=10 \%$ with an isotropic microstructure. The isotropic measurements that are shown in Fig. 3 are obtained when the suspension is mixing by hand-agitation and can be compared to the anisotropic measurements obtained with sheared suspensions shown in Fig. 4. The structure factors from sheared suspensions shown in Fig. 4(b) are smoother when compared to the structures factors from suspensions mixed by hand-agitation, as expected. Indeed, the structure factors were computed by averaging over $1000 \mathrm{rf}$ signals for sheared suspensions, versus $200 \mathrm{rf}$ signals for suspensions mixed by hand due to the difficulty of performing the experiments. Even in the presence of steric repulsion and particle roughness, there is no favorite pair trajectories because of the random motion of the particle resulting from the mixing by hand-agitation. The pair-correlation function and the structure factor from hard spheres depend only on the particle radius and the volume fraction, as described by the Percus-Yevick approximation. ${ }^{20}$ That is why the $B S C_{\text {meas }}$ and $S_{\text {meas }}$ measured from suspensions mixed by hand-agitation were satisfactorily predicted by the SFM modeling (see Fig. 3).

\section{Comparison between simulations and experiments}

The comparison between simulations and experiments can be only qualitative because the particle roughness in simulations $\left(\epsilon_{R} \approx 3 \times 10^{-3} a\right)$ differs from those of particles used in ultrasonic experiments $\left(\epsilon_{R} \approx 16 \times 10^{-3} a\right)$. Moreover, an important difference is the Reynolds numbers: $R e=0$ for simulations, against $\mathrm{Re}=1$ for ultrasonic experiments. A higher Reynolds number modifies the probability density function and the pair correlation function, increasing their tail-like zone in the recession quadrant. ${ }^{25}$ We may assume that the difference between numerical and experimental structural factors are mainly due to the different Reynolds number. Future studies should focus on experiments combining ultrasonic measurements and optical non-intrusive techniques, such as the refractive index matching technique, ${ }^{15,23}$ to study the same suspensions and allow quantitative comparison. The suspending fluid using the refractive index matching technique has generally a very high viscosity (for example, $\eta=0.85$ Pa.s in Blanc et al. ${ }^{15}$ ). From the ultrasonic point of view, the major difficulty to conduct these studies is to evaluate accurately the absorption of viscous suspensions because the compensation for absorption will greatly modify the frequency-dependence of $B S C_{\text {meas }}$ (and thus of $S_{\text {meas }}$ ) for highly viscous suspension. Therefore, in the present study, ultrasonic experiments were conducted on concentrated suspensions with suspending fluid having low viscosity $(\eta=7.7$ mPa.s $)$ and thus low absorption of ultrasound.

\section{Open questions for future research on deformable particle suspensions}

The present study demonstrates that the angulardependent BSCs and structure factors are caused by the shear-induced anisotropic microstructure within the suspensions. One could question if this anisotropic microstructure can occur in concentrated suspensions of deformable particles such as red blood cells since quantitative ultrasound techniques based on parameterizing the BSC are largely used for the characterization of red blood cell aggregation under flow condition. ${ }^{1,2}$ The rheology of concentrated suspensions of deformable particles and its connection to the suspension microstructure is quite unexplored (see subsection 7.5 of Ref. 24). This is mainly due to the experimental difficulty in determining the spatial arrangement within concentrated opaque suspensions. The ability of quantitative ultrasound to measure the structure factors may prove valuable in the future in this field.

Some experiments ${ }^{26,27}$ have been performed to measure the angle dependent BSCs in sheared concentrated suspensions of aggregating red blood cells $(\Phi=40 \%)$. The BSC results given in Fig. 3 of Ref. 27 are based on the same experimental set-up that combines Couette flow device and ultrasonic transducer. In this preliminary study, the angulardependent BSCs averaged over the $15-20 \mathrm{MHz}$ bandwidth are not straightforward to interpret (see Fig. 3 in Ref. 27) since it can originate from both the flow-aligned aggregate structure and the sheared-induced anisotropic microstructure of effective scatterers (i.e., aggregates). However, the highest shear rate of $140 \mathrm{~s}^{-1}$ is likely to produce a suspension of disaggregated red blood cells. This suggests the microstructure that develops under simple shear in this suspension happens to be anisotropic. Note that this anisotropic behavior cannot originate from the biconcave shape of red blood cells since the scattering from a single red blood cell is isotropic at low frequencies $<20 \mathrm{MHz}$ (i.e., in the Rayleigh scattering regime). Further ultrasonic studies need to be conducted on concentrated suspensions of disaggregated red blood cells for a better understanding of how the formation of anisotropic microstructure influences the BSC amplitude. More specifically, it would be of great interest to compare the angular-dependent BSCs in simple shear flow (Couette flow) 
versus Poiseuille flow, since Poiseuille flow is closer to the in vivo situation and one could expect that the shearedinduced anisotropic microstructure is less pronounced in tubular flow with variable shear.

\section{E. Concluding remarks}

We performed ultrasonic experiments in a concentrated suspension of non-Brownian particles to investigate how the shear-induced anisotropic microstructure affects the ultrasonic backscattering measurements (i.e., the BSC and the structure factor). For all the studied volume fractions, the measured structure factor has been shown to be anisotropic with axial symmetry in the orthogonal direction of the angular position of the depleted area of the pair correlation function. The experimental structure factors are in good agreement with the structure factors determined from Stokesian dynamics numerical simulations. Therefore, sheared concentrated suspensions cannot be modeled as isotropic media since shear-induced anisotropic microstructure greatly affect the frequency dependence and amplitude of BSC and structure factor. If the parameterization of the BSC is used to estimate the local scatterer size and/or volume fraction, the assumption of isotropic media would obviously create a bias against the parameter estimation. These results are of major interest in the field of ultrasonic characterization of opaque suspensions, such as blood or industrial materials (food, cosmetics), for which the measures are performed under shear conditions.

\section{ACKNOWLEDGMENTS}

We would like to thank Alain Ciffreo from INPHYNI UMR 7010 for providing the Stokesian dynamics simulation data, Bloen Metzger from IUSTI UMR 7343 for helping in designing the Couette flow device, as well as P. Cervetti, S. Noel from IUSTI UMR 7343 and V. Long from LMA UMR 7031 for building the experimental set up. This work was supported by the French National Research Agency (Grant No. ANR-15-CE19-0017), and the Labex MEC (Grant No. ANR-10-LABX-0092).

${ }^{1}$ G. Cloutier and Z. Qin, "Ultrasound backscattering from non-aggregating and aggregating erythrocytes-A review," Biorheology 34(6), 443-470 (1997).

${ }^{2}$ E. Franceschini and G. Cloutier, "Modeling of ultrasound backscattering by aggregating red blood cells," in Quantitative Ultrasound in Soft Tissues (Springer, New York, 2013), pp. 117-145.

${ }^{3}$ L. Elvira, P. Vera, F. J. Cañadas, S. K. Shukla, and F. Montero, "Concentration measurement of yeast suspensions using high frequency ultrasound backscattering," Ultrasonics 64, 151-161 (2016).

${ }^{4}$ J. E. Carlson, J. Stener, A. Sand, and B. I. Pålsson, "Monitoring local solids fraction variations in multiphase flow using pulse-echo ultrasound," Phys. Procedia 70, 376-379 (2015).

${ }^{5}$ J. F. Stener, J. E. Carlson, A. Sand, and B. I. Pålsson, "Monitoring mineral slurry flow using pulse-echo ultrasound," Flow Meas. Inst. 50, 135-146 (2016).

${ }^{6}$ B. Saint-Michel, H. Bodiguel, S. Meeker, and S. Manneville, "Simultaneous concentration and velocity maps in particle suspensions under shear from rheo-ultrasonic imaging," Phys. Rev. Appl. 8(1), 014023 (2017).
${ }^{7}$ M. L. Oelze and J. Mamou, "Review of quantitative ultrasound: Envelope statistics and backscatter coefficient imaging and contributions to diagnostic ultrasound," IEEE Trans. Ultrason. Ferroelectr. Freq. Control 63(2), 336-351 (2016).

${ }^{8}$ D. Savery and G. Cloutier, "A point process approach to assess the frequency dependence of ultrasound backscattering by aggregating red blood cells," J. Acoust. Soc. Am. 110(6), 3252-3262 (2001).

${ }^{9}$ E. Franceschini, R. K. Saha, and G. Cloutier, "Comparison of three scattering models for ultrasound blood characterization," IEEE Trans. Ultrason. Ferroelectr. Freq. Control 60(11), 2321-2334 (2013).

${ }^{10} \mathrm{~V}$. Twersky, "Low-frequency scattering by correlated distributions of randomly oriented particles," J. Acoust. Soc. Am. 81(5), 1609-1618 (1987).

${ }^{11}$ L. Haider, P. Snabre, and M. Boynard, "Rheo-acoustical study of the shear disruption of reversible aggregates. Ultrasound scattering from concentrated suspensions of red cell aggregates," J. Acoust. Soc. Am. 107(3), 1715-1726 (2000).

${ }^{12} \mathrm{~F}$. T. Yu and G. Cloutier, "Experimental ultrasound characterization of red blood cell aggregation using the structure factor size estimator," J. Acoust. Soc. Am. 122(1), 645-656 (2007).

${ }^{13}$ E. Franceschini, F. T. Yu, F. Destrempes, and G. Cloutier, "Ultrasound characterization of red blood cell aggregation with intervening attenuating tissue-mimicking phantoms," J. Acoust. Soc. Am. 127(2), 1104-1115 (2010).

${ }^{14}$ R. de Monchy, J. Rouyer, F. Destrempes, B. Chayer, G. Cloutier, and E. Franceschini, "Estimation of polydispersity in aggregating red blood cells by quantitative ultrasound backscatter analysis," J. Acoust. Soc. Am. 143(4), 2207-2216 (2018).

${ }^{15}$ F. Blanc, E. Lemaire, A. Meunier, and F. Peters, "Microstructure in sheared non-brownian concentrated suspensions," J. Rheol. 57(1), 273-292 (2013).

${ }^{16}$ S. Deboeuf, N. Lenoir, D. Hautemayou, M. Bornert, F. Blanc, and G. Ovarlez, "Imaging non-Brownian particle suspensions with $\mathrm{x}$-ray tomography: Application to the microstructure of newtonian and viscoplastic suspensions," J. Rheol. 62(2), 643-663 (2018).

${ }^{17}$ J. J. Faran, Jr., "Sound scattering by solid cylinders and spheres," J. Acoust. Soc. Am. 23(4), 405-418 (1951).

${ }^{18} \mathrm{E}$. Franceschini and R. Guillermin, "Experimental assessment of four ultrasound scattering models for characterizing concentrated tissuemimicking phantoms," J. Acoust. Soc. Am. 132(6), 3735-3747 (2012).

${ }^{19}$ E. Franceschini, R. de Monchy, and J. Mamou, "Quantitative characterization of tissue microstructure in concentrated cell pellet biophantoms based on the structure factor model," IEEE Trans. Ultrason. Ferroelectr. Freq. Control 63(9), 1321-1334 (2016).

${ }^{20} \mathrm{M}$. Wertheim, "Exact solution of the percus-yevick integral equation for hard spheres," Phys. Rev. Lett. 10(8), 321-323 (1963).

${ }^{21}$ L. X. Yao, J. A. Zagzebski, and E. L. Madsen, "Backscatter coefficient measurements using a reference phantom to extract depth-dependent instrumentation factors," Ultrason. Imaging 12(1), 58-70 (1990).

${ }^{22}$ A. Han and W. D. OBrien, "Structure function for high-concentration biophantoms of polydisperse scatterer sizes," IEEE Trans. Ultrason. Ferroelectr. Freq. Control 62(2), 303-318 (2015).

${ }^{23}$ F. Blanc, F. Peters, and E. Lemaire, "Experimental signature of the pair trajectories of rough spheres in the shear-induced microstructure in noncolloidal suspensions," Phys. Rev. Lett. 107(20), 208302 (2011).

${ }^{24}$ É. Guazzelli and O. Pouliquen, "Rheology of dense granular suspensions," J. Fluid Mech. 852, 1-73 (2018).

${ }^{25} \mathrm{H}$. Haddadi and J. F. Morris, "Microstructure and rheology of finite inertia neutrally buoyant suspensions," J. Fluid Mech. 749, 431-459 (2014).

${ }^{26} \mathrm{C}$. Guilbert, F. Yu, and G. Cloutier, "New observations on the anisotropy of ultrasound blood backscatter as a aunction of frequency and shear rate," in Proceedings of the 2007 IEEE International Ultrasonics Symposium (IUS), New York (October 28-31, 2007), pp. 1013-1016.

${ }^{27}$ J. Rouyer, L. Chinchilla, O. Lombard, and E. Franceschini, "Characterizing the erythrocyte aggregation using the anisotropy of ultrasonic backscatter," in Proceedings of the 2018 IEEE International Ultrasonics Symposium (IUS), Kobe, Japan (October 22-25, 2018), pp. $1-4$.

${ }^{28}$ See supplementary material at https://doi.org/10.1121/10.0000803 for a figure showing the angular variation of Smeas $(k a=0.8)$ obtained from the two series of experiments. 